\title{
Systems Engineering in der Produktentwicklung: Verständnis, Theorie und Praxis aus ingenieurswissenschaftlicher Sicht
}

\author{
Tobias Huth ${ }^{1}$ Thomas Vietor ${ }^{1}$ \\ Online publiziert: 6. Februar 2020 \\ (c) Der/die Autor(en) 2020
}

Heutige Produkte vereinen in sich eine Vielzahl von Elementen unterschiedlicher Disziplinen, um ihre Funktionalitäten $\mathrm{zu}$ realisieren und dem Nutzer bereitzustellen. Beispielsweise war eine Küchenmaschine früher ein ,einfaches" Produkt aus mechanischen und wenigen elektronischen Bauteilen. Die einzige Aufgabe der elektronischen Bauteile war es in der Regel einzig den Knethaken oder Quirl mit wenigen unterschiedlichen Geschwindigkeitsstufen in Rotation zu versetzt. Aktuelle Küchenmaschinen bieten den Nutzern heute deutlich mehr Funktionalitäten, wie spezielle Knet-Programme mit Intervallen von linksund rechtsläufigen Rotationen, unterschiedliche Gar-Programme oder eingebaute Waagen. Diese Entwicklung lässt sich bei vielen Produkten in ähnlicher Form beobachten. So hat sich die Anzahl von Steuergeräten in heutigen Fahrzeugen massiv erhöht, die Anzahl der Code-Zeilen ist alleine in den letzten Jahren um rund das fünfzehnfache gestiegen und der größte Teil der Innovationen im Automobilbereich entstehen heute im Bereich der Software und Elektronik (Wilms et al. 2019).

Produkte wie Fahrzeuge oder Küchenmaschinen sind technische Systeme. Ein System setzt sich dabei aus einer Reihe unterschiedlicher Elemente aus unterschiedlichen Disziplinen (bspw. Mechanik, Elektrik/Elektronik, Software) zusammen, die untereinander in Beziehung stehen. Die zuvor erläuterte Funktionszunahme heutiger Produkte korreliert dabei mit einer allgemeinen Zunahme der Komplexität heutiger Produkte. Die Steigerung der Komplexität resultiert dabei aus einer gleichzeitigen Zunahme der Anzahl unterschiedlicher Elemente als auch der unterschiedlichen Beziehungen zwischen den Elementen. Die

Tobias Huth

tobias.huth@tu-braunschweig.de

Prof. Dr.-Ing. Thomas Vietor

t.vietor@tu-braunschweig.de

1 Institut für Konstruktionstechnik, Technische Universität Braunschweig, Hermann-Blenk-Straße 42, 38108 Braunschweig, Deutschland
Komplexitätszunahme innerhalb der Produkte geht mit einer Steigerung der Komplexität im Produktentwicklungsprozess einher, da bspw. der Abstimmungs- und Kommunikationsbedarf zwischen den beteiligten Disziplinen steigt und Informationen ausgetauscht werden müssen. Zur Handhabung der steigenden Komplexität der Produkte und der Entwicklungsprozesse werden zunehmend Methoden des Systems Engineerings in Unternehmen eingeführt.

Im Folgenden wird ein kurzer Überblick über die Entwicklung des Systems Engineerings (SE) und der dem SE zugrundeliegenden systemtheoretischen Grundlagen gegeben. Darauf aufbauend werden Definitionen des SE aufgezeigt, eine Auswahl etablierter SE-Ansätze vorgestellt und die Weiterentwicklung des Systems Engineerings zum Model-based Systems Engineerings dargestellt.

\section{Systemtheoretische Grundlagen und Begriffe}

Ein System ist nach ROPOHL eine Darstellungsform, durch die ein Ausschnitt der Wirklichkeit abgebildet wird. Die Wirklichkeit wiederum wird vom Menschen in ,perspektivische Konstruktionen und Interpretationen“ (Ropohl 2012) untergliedert. Was bedeutet, dass der Mensch die Wirklichkeit aus seiner subjektiven Sicht in Systeme, also Zusammenhangsgeflechte, einteilt. Die Zusammenhänge zwischen einigen Elementen sind dabei nachweisbar vorhanden, zwischen anderen jedoch nicht. Elemente, zwischen denen ein Zusammenhang eindeutig identifizierbar ist, werden nach ROPOHL als Ganzheit bezeichnet. Diese Ganzheiten sind die Untersuchungsobjekte der allgemeinen Systemtheorie (Ropohl 1975). Durch die Systemtheorie werden aus diesen Ganzheiten Systeme konstruiert, die den für eine Betrachtung relevanten Teil der Ganzheit umfassen (Ropohl 2012).

Systeme sind keine natürlichen Gebilde, sondern Modelle des menschlichen Denkens (Ropohl 2012). Hiermit wird die allgemeine Systemtheorie zu einer ,operativen Theorie über die Art und Weise, wie man Modelle von 
beliebigen ganzheitlichen Erfahrungsbereichen konstruiert" (Ropohl 2012).

Hieraus folgt, dass im Sinne der allgemeinen Systemtheorie ein System ein Modell einer Ganzheit ist. „Ein System bildet eine Ganzheit ab, berücksichtigt dabei aber nur ausgewählte Eigenschaften der Ganzheit und ist nur für einen Teil von Menschen eine gewisse Zeitlang für bestimmte Formen des Denkens und Handelns relevant" (Ropohl 2012). Ein solches Modell bzw. System muss einer kritischen Prüfung standhalten können, nur dann kann es den gewünschten Zweck erfüllen. Die allgemeine Systemtheorie führt drei formale Konzepte für Systeme ein, die jeweils unterschiedliche Inhalte abbilden und sich damit für unterschiedliche Zielsetzungen eignen:

- funktionales Systemkonzept: Das eigentliche System wir als Black Box aufgefasst. Lediglich die Ein- und Ausgänge des Systems sind bekannt bzw. von außen ersichtlich. Als Funktion des Systems wird die (reproduzierbare) Beziehung bezeichnet, die das System zwischen den Einund Ausgängen herstellt. „Das funktionale Systemdenken sieht ausdrücklich von der materiellen Konkretisierung und vom inneren Aufbau des Systems ab und beschränkt sich auf das Verhalten einer Ganzheit in ihrer Umgebung" (Ropohl 2012).

- strukturales Systemkonzept: Bei diesem Konzept wird ein System als Menge unterschiedlicher Elemente, zwischen denen eine Vielzahl von Relationen bestehenden können, aufgefasst. „Dem strukturalen Ansatz geht es zum einen um die Vielfalt möglicher Beziehungsgeflechte, die in einer gegebenen Menge von Elementen bestehen und dadurch unterschiedliche Systemeigenschaften hervorrufen können. Zum anderen befasst es sich auch mit der Beschaffenheit der Elemente, von der es abhängt, wie gut sie sich in ein System integrieren lassen“ (Ropohl 2012). Beim strukturalen Systemkonzept werden die einzelnen Elemente somit nicht für sich alleine, sondern in ihrem Kontext und Zusammenspiel mit anderen Elementen betrachtet.

- hierarchisches Systemkonzept: Dieses Systemkonzept dient dazu unterschiedliche Abstraktions- bzw. Konkretisierungsebenen abzubilden. Denn einerseits kann ein betrachtetes System (A) wiederum aus Elementen, die für sich betrachtet ebenfalls Systeme sind (Aus Sicht von A sind es dessen Subsysteme), bestehen. Andererseits kann das System A selbst Teil eines übergeordneten Systems (Aus Sicht von A ist es dessen Supersystem) sein. Je nachdem in welche Richtung die Betrachtung mittels des hierarchischen Systemkonzeptes erfolgt, rücken die Details oder die größeren Zusammenhänge in den Fokus der Betrachtung. „Das Systemdenken [ist] für beide Erkenntnisstrategien offen, für die immer tiefer greifende Analyse von Einzelheiten ebenso wie für die immer wei- ter greifende Synthese von Zusammenhängen“ (Ropohl 2012).

Die zuvor genannten Systemkonzepte schließen sich dabei nicht untereinander aus, sondern können auch miteinander verbunden werden, um Systeme mit einer bestimmten Zielsetzung zu beschreiben.

\section{Was zeichnet Systems Engineering aus?}

Die drei zuvor erläuterten Systemkonzepte und die Begriffe des Systems und des Modells spielen im Kontext des Systems Engineerings eine wichtige Rolle. Der Begriff Systems Engineering selbst und die Frage was er eigentlich umfasst können aber nicht abschließend definiert werden.

Der INTERNATIONAL COUNCIL OF SYSTEMS ENGINEERING (INCOSE) definiert Systems Engineering wie folgt: „Systems Engineering (SE) ist ein interdisziplinärer Ansatz zur Unterstützung der Realisierung von erfolgreichen Systemen. SE fokussiert darauf, die Kundenbedürfnisse und die geforderte Funktionalität möglichst früh im Entwicklungsprozess zu definieren, die Anforderungen zu dokumentieren und dann mit dem Systementwurf und der Systemvalidierung fortzufahren, während dabei zugleich das gesamte Problem im Blick behalten wird, einschließlich der Verwendung, der Kosten und dem Zeitplan, der Leistungswerte, der Schulungsmaßnahmen und der Unterstützungsmaßnahmen, der Nachweise und Zertifizierungen, der Herstellung und der Entsorgung des Systems. Durch SE werden alle Fachdisziplinen in einem Teamansatz zusammengefasst, in dem ein strukturierter Entwicklungsprozess vom Konzept über die Herstellung bis zur Verwendung des Systems bechrieben wird. Systems Engineering betrachtet sowohl die wirtschaftlichen als auch die technisches Bedarfe aller Kunden, mit dem Ziel, ein qualitativ hochwertiges Produkt zu schaffen, das den Bedarf des Kunden gerecht wird.“ (Walden et al. 2017). Die Definition fasst sehr kompakt viele Merkmale des Systems Engineerings zusammen. Ein wesentliches Merkmal des Systems Engineerings ist die Interdisziplinarität. Im Sinne des Systems Engineerings arbeiten wenigstens die Disziplinen Mechanik, Elektrik/Elektronik und Informatik/Softwareentwicklung während der Entwicklung technischer Systeme als Team zusammen. Die Fokussierung auf den Kunden ist ein weiteres wesentliches Merkmal. Im Sinne des Systems Engineerings beginnt die Produktentwicklung mit der Erfassung der vom Kunden gestellten Anforderungen in Bezug auf das spätere Produkt und dessen Funktionalitäten. Ausgehend davon werden die Anforderungen anderer Anspruchsgruppen an das Produkt sowie Randbedingungen, die sich aus dem möglichen Einsatzorten ergeben, systematisch erfasst. Bezogen auf das Beispiel der Küchenmaschine sind mögliche 
Funktionalitäten eine integriete Waage oder unterschiedliche Garprogramme mit definierten Temperatur- und Gewinschwindigkeitsprofilen des Quirls. Als Randbedingungen sind gebräuchliche Oberflächeneigenschaften von Küchenarbeitsplatten zu berücksichtigen, um auch bei hohen Rühergeschwindigkeiten einen sicheren Stand der Küchenmaschine zu gewährleisten. Im weiteren Verlauf der Entwicklung sind diese Anforderungen wichtiger Input für die einzelnen Entwicklungsaktivitäten und dienen während der Realisierung des Produktes als Basis für durchzuführende Tests während der Validierung des Produktes, um sicherzustellen, dass das entwickelte Produkt den Anfangs ermittelten Anforderungen entspricht und die Kundenbedürfnisse erfüllt.

Systems Engineering kann auch als eine Meta-Disziplin, die u.a. den Informationsfluss zwischen den einzelnen Disziplinen (bspw. Software-, Elektronik- und MechanikEntwicklung) durch die Bereitstellung geeigneter Schnittstellen und Prozesse unterstützt und die Entwicklung der unter den gesetzten Randbedingungen bestmöglichen Lösung zum Ziel hat, verstanden werden (Weilkiens 2014; Friedenthal et al. 2015). Um dieses Ziel zu erreichen werden im Systems Engineering nicht nur die reine technische Entwicklung, sondern auch Aspekte des Projektmanagements im Zusammenspiel berücksichtigt (Haberfellner et al. 2019).

Durch die zuvor genannte Definition und die weiteren Aspekte wird letztlich ein sehr großer Definitionsraum für das Systems Engineering aufgespannt, in dem sich viele unterschiedlichste Ansätze, Methoden und Prozesse verorten lassen. Um die Vielfalt der möglichen Ansätze im Systems Engineering kurz aufzuzeigen, werden im folgenden drei beispielhafte Ansätz und ihre mögliche Anwendung im Kontext des Beispiels der Küchenmaschine genannt.

Mit einem Ansatz zur modellbasierten Beschreibung des zu entwickelnden Systems (Inkermann et al. 2019) können für eine Küchenmaschine, ausgehend von den Kundenbedüfnissen, Funktionalitäten abgeleitet und detailliert beschrieben werden. Darauf aufbauend wird die Struktur der Küchenmaschine, also die für die Umsetzung der Funktionalitäten erforderlichen Elemente aus den Disziplinen Mechanik, Elektronik oder Software sowie deren Verknüpfungen beschrieben. Für die Küchenmaschine bedeutet dies bspw. dass sie einen Motor hat, der mit einer Steuerung verbunden ist und der Quirl über eine Kupplung mit dem Motor verbunden wird. Diese drei Elemente tragen zusammen dazu bei Garprogramm mit den Geschwindigkeitsprofilen des Quirls zu realisieren.

Darüber hinaus können mit Ansätzen zur Beschreibung der Konfigurationsräume von Produktlinien (Huth et al. 2017) bspw. Kombinationsmöglichkeiten von Zusatzgeräten und unterschiedlichen Generationen/Varianten einer Küchenmaschine abgebildet werden. Weitere Systems En- gineering Ansätze betrachten die Entwicklungsorganisation im Unternehmen als System und versuchen dieses mit seinen Elementen (Prozesse, Rollen, Menschen, Produktinformationen und Werkzeuge) zu beschreiben und damit die Einführung der zuvor genannten Systems Engineering Ansätze in Unternehmen zu unterstützen (Huth et al. 2018).

Systems Engineering ist somit keine spezielle (Produktentwicklungs-)Methode sondern entwickelt sich durch die Vielfältigkeit der unterschiedlichen Ansätze mehr und mehr zu einer eigenständigen Disziplin (Gausemeier et al. 2015; Walden et al. 2017).

\section{Model-based Systems Engineering als Weiterentwicklung des Systems Engineerings}

Um den Unzulänglichkeiten früherer dokumentenbasierter Systems Engineering Ansätze (wie bspw. mangelnder Nachverfolgbarkeit von Abhängigkeiten zwischen Anforderungen und Systemelementen sowie Entscheidungen während der Entwicklung) zu begegnen, wurden und werden Ansätze zum sog. modellbasierten Systems Engineering (engl. Model-based Systems Engineering (MBSE)) entwickelt (Alt 2012). Ziel des Model-based Systems Engineerings ist es, die Ergebnisse verschiedener Entwicklungsaktivitäten in einem zentralen gemeinsamen Systemmodell $\mathrm{zu}$ dokumentieren und kontextspezifische Sichten (bspw. um die folgenden Fragen während der Produktentwicklung $\mathrm{zu}$ beantworten ,Welche Kundenanforderungen sind für die Entwicklung der Steuerung der Küchenmaschine wichtig?“ oder „Welche Elemente der Maschine sind für die Funktionalität der integrierten Waage relevant und müssen bei einer Erhöhung des Messbereichs ggf. ausgetauscht werden?") dieser Informationen und ihrer Abhängigkeiten generieren zu können. Das Model-based Systems Engineerings fordert daher den Übergang von heterogenen, dokumentenbasierten Produktmodellen zu konsistenten und vernetzten Produktmodellen. Die beteiligten Disziplinen nutzen die Modelle dabei als Informationsquelle und -senke, um ihre Arbeitsergebnisse zu dokumentieren und mit anderen Modellelementen in Beziehung zu setzen (Alt 2012). Die Modellierung des Systems ist für das Modelbased Systems Engineerings von grundlegender Bedeutung. Für diese Modellierung werden auch wieder die Grundsätze der Systemtheorie angewandt. Die Modellierung basiert dabie auf den drei Elementen Sprache, Werkzeug und Methode. Die Methoden geben dabei in der Regel durch ein systematisches Vorgehen sowie eine Festlegung der im Modell abzubildenen Aspekte des technisches Systems (vgl. Ansätze im vorherigen Abschnitt) einen Rahmen für die Modellierung vor. Bestehende Standards für Modellierungssprachen wie UML (Object Management Group 
2017b) oder SysML (Object Management Group 2017a) definieren die zu verwendenden Semantik und Syntax, und bilden im Rahmen der Methode die formale Grundlage für die Modellierung. Mittels in der Regel softwarebasierte Modellierungswerkzeuge werden die Modelle unter Einhaltung der Semantik und Syntax erstellt und Analysen (wie bspw. die Beziehung zwischen Kundenanforderungen und der Steuerung der Küchenmaschine) durchgeführt. Die im Model-based Systems Engineering verwendeten Sprachen sind dabei generischer Natur, um die Darstellung verschiedenartiger Systeme (z.B. Küchenmaschinen und Fahrzeugsysteme) gleichermaßen zu unterstützen.

Das Model-based Systems Engineering formalisiert das Systems Engineering durch den Einsatz von Modellen, um unter anderem folgende Ziele zu unterstützen (Borky und Bradley 2019):

- Sicherstellung von Genauigkeit, Wiederholbarkeit und Herstellbarkeit in Systems Engineering Prozessen (bspw. sichert die Nachvollziehbarkeit der Entscheidung während der Entwicklung die Genauigkeit und Wiederholbarkeit)

- Förderung von Qualität, Vollständigkeit und Korrektheit im Systemdesign (bspw. fördert die Nachverfolgbarkeit/ Zuordnung von Anforderungen zu adressierten Elementen des Produktes die Korrektheit im Systemdesign)

- Risikominimierung bei der Anforderungsanalyse, dem Design, der Integration und dem Testen sowie bei anderen Aktivitäten (bspw. reduziert die Ableitung von relevanten Testfällen auf Basis der Kundenanforderungen das Risiko das Produkt um Kundenbedürfnis vorbei zu entwickeln)

- Ausbau der Kommunikation und Synchronisierung von Aktivitäten quer durch die Organisations und Disziplinen (bspw. können mögliche Änderungsbedarfe für die Elemente der anderen Disiplinen aus den Modellen abgeleitet werden und die gemeinsame Entwicklung von Alternativen initiiert werden)

Im Kern ist das Model-based Systems Engineering somit die konsequente Kombination des Systems Engineerings mit den Grundsätzen der modellbasierten objekt-orientierten Softwareentwicklung und bietet damit zusätzliche Möglichkeiten um die Entwicklung komplexer Systeme hanhabbar zu machen.

\section{Systems Engineering in der Praxis}

Um zu klären, wie Systems Engineering in der industriellen Praxis angekommen ist und eingesetzt wird wurden sowohl Systems Engineering Experten als auch Führungskräfte ohne explizite Systems Engineering Kenntnisse befragt (Gausemeier et al. 2015). Zu den mit Systems Engineering as- soziierten Schlüsselbegriffen gehören u.a. Ganzheitliches Systemverständnis, Von der Anforderung bis zur Systementwicklung und Interdisziplinärer Ansatz (Gausemeier et al. 2015). Beim in der Industrie vorherrschende Systemverständnis scheint oft einzig das zu entwickelnde System auf eine systemische Art betrachtet zu werden. Das zu entwickelnde System kann aber natürlich auch in Zusammenhang mit weiteren „Systemen“, wie dem Unternehmen, dem Entwicklungsprojekt oder dem sozioökonomischen Umfeld stehen (Gausemeier et al. 2015). Aus der Studie lässt sich aber unter anderem zusammenfassen, dass in der industriellen Praxis in vielen Fällen ein interdisziplinärer Ansatz zur ganzheitlichen Entwicklung von Systemen unter dem Terminus System Engineering verstanden wird.

Neben dem Verständnis beleuchtet die genannte Studie auch die Erwartungen an Systems Engineering seitens der befragten Industrievertreter. Hier wurden unter anderem als erwartete Nutzen die Koordination der disziplinübergreifenden Zusammenarbeit, die Berücksichtigung aller Stakeholder und verbesserte Planung und Steuerung der Entwicklungsprojekte genannt (Gausemeier et al. 2015). Diesen erhofften Nutzen stehen aber natürlich auch Hindernisse gegenüber. $\mathrm{Zu}$ den am meisten genannten Hindernissen zählen die schlechte bis unmögliche Quantifizierbarkeit des Nutzens von Systems Engineering Ansätzen, die mangelnde Verfügbarkeit von Einführungsmethoden für Systems Engineering Ansätze und die fehlenden Kompetenzen bei den Mitarbeitern (Gausemeier et al. 2015). Letztere machen umfangreiche Weiterbildungsmaßnahmen bei der und für die Einführung von Systems Engineering in Unternehmen nötig. Kompetenzen gilt es dabei an Prozesse gekoppelt zu betrachten (Kauffeld und Paulsen 2018; zur nachhaltigen Weiterbildung vgl. Kauffeld 2016). In der Studie wird herausgestellt, dass gerade auch die heute angebotenen Studiengänge die Bedürfnisse der Industrie nach Systems Engineering Kompetenzen durch ihre in der Regel fachdisziplinspezifische Ausrichtung nicht abdecken können (Gausemeier et al. 2015).

Die Autoren der Studie kommen bezüglich des Einsatzes und Verständnisses von Systems Engineering in der Industrie zu folgendem Resümee: „Im deutschsprachigen Raum ist die Verbreitung von Systems Engineering stark branchenabhängig. So ist Systems Engineering in der Luft- und Raumfahrttechnik fest etabliert. In der Fahrzeugindustrie wird SE inzwischen als Befähiger gesehen, es gewinnt zunehmend an Bedeutung und wird von den OEMs vorangetrieben. Aber in der Breite, insbesondere dem in Deutschland stark mittelständisch geprägten Maschinen- und Anlagenbau, wird Systems Engineering trotz seiner hohen Bedeutung nicht eingesetzt." (Gausemeier et al. 2015) Hieraus folgt, dass es gerade Bezüglich der Einführung von Systems Engineering in den Unternehmen und auch der Transformation etablierter, oftmals unidisziplinärer, Entwicklungs- 
strukturen hin zu interdisziplinär ausgerichteten Entwicklungsorganisationen Unterstützungsbedarf gibt. Dieser Bedarf selbst kann nur durch interdisziplinäre Forschungsarbeiten der Arbeits- und Ingenieurswissenschaften adressiert werden, da in den Unternehmen vorhandenen Strukturen reorganisiert, Kompetenzen der Mitarbeiter gezielt erweitert und neue Entwicklungsmethoden eingeführt werden müssen.

\section{Zusammenfassung}

Systems Engineering an und für sich ist ein eher unscharfer Begriff, es können darunter sowohl einzelne (Entwicklungs-)Methoden, Prozesse, die Weiterentwicklung Model-based Systems Engineering aber auch eine ganze Disziplin verstanden werden. Diese Breite sollte immer mitberücksichtigt werden, wenn der Begriff Systems Engineering genutzt wird. Dennoch beruht das dem Systems Engineering zugrundeliegende Verständnis immer auf dem Denken in Systemen und der Berücksichtigung funktionaler, strukturaler und hierarchischer Aspekte. Systems Engineering wird gerade auch in der Industrie als interdisziplinärer ganzheitlicher Entwicklungsansatz gesehen, der die Einbeziehung aller Stakeholder und die Handhabung der weiter zunehmenden Komplexität unterstützt. Systems Engineering bietet das Potential bei der Entwicklung zukünftiger komplexer Produkte eine wichtige Rolle einzunehmen und diese unter Umständen erst zu ermöglichen, da etablierte Entwicklungsmethoden und -prozesse die u. a. aus der starken Vernetzung der einzelnen Elemente resultierende Komplexität nicht mehr ausreichend handhaben können. Diese Potentiale können aber nur durch den Aufbau der benötigten Kompetenzen bei den Mitarbeitern, sowohl durch entsprechende Ausbildungsaber insbesondere auch durch Weiterbildungsmaßnahmen, gehoben werden.

Funding Open Access funding provided by Projekt DEAL.

Open Access Dieser Artikel wird unter der Creative Commons Namensnennung 4.0 International Lizenz veröffentlicht, welche die Nutzung, Vervielfältigung, Bearbeitung, Verbreitung und Wiedergabe in jeglichem Medium und Format erlaubt, sofern Sie den/die ursprünglichen Autor(en) und die Quelle ordnungsgemäß nennen, einen Link zur Creative Commons Lizenz beifügen und angeben, ob Änderungen vorgenommen wurden.

Die in diesem Artikel enthaltenen Bilder und sonstiges Drittmaterial unterliegen ebenfalls der genannten Creative Commons Lizenz, sofern sich aus der Abbildungslegende nichts anderes ergibt. Sofern das betreffende Material nicht unter der genannten Creative Commons Lizenz steht und die betreffende Handlung nicht nach gesetzlichen Vorschriften erlaubt ist, ist für die oben aufgeführten Weiterverwendungen des Materials die Einwilligung des jeweiligen Rechteinhabers einzuholen.
Weitere Details zur Lizenz entnehmen Sie bitte der Lizenzinformation auf http://creativecommons.org/licenses/by/4.0/deed.de.

\section{Literatur}

Alt, O. (2012). Modellbasierte Systementwicklung mit SysML. München: Carl Hanser.

Borky, J. M., \& Bradley, T.H. (2019). Effective model-based systems engineering. Cham: Springer.

Friedenthal, S., Moore, A., \& Steiner, R. (2015). A practical guide to SysML: The systems modeling language (3. Aufl.). The MK/OMG Press. Burlington: Morgan Kaufmann.

Gausemeier, J., Dumitrescu, R., Steffen, D., Czaja, A., Wiederkehr, O., \& Tschirner, C. (2015). Systems Engineering in der industriellen Praxis. https://www.iem.fraunhofer.de/content/dam/iem/de/ documents/Studie\%20Systems\%20Engineering_deutsch.pdf. Zugegriffen: 25. Febr. 2019.

Haberfellner, R., de Weck, O., Fricke, E., \& Vössner, S. (2019). Systems engineering. Cham: Springer.

Huth, T., Inkermann, D., \& Vietor, T. (2017). Ein Ansatz für eine integrierte modellbasierte Anforderungs- und Variantenmodellierung. In J. Gausemeier, E. Bodden, F. Dressler, R. Dumitrescu, F. Meyer auf der Heide, C. Scheytt \& A. Trächtler (Hrsg.), Wissenschaftsforum Intelligente Technische Systeme (WInTeSys). Paderborn, 11.-12. Mai 2017. (S. 169-182). Paderborn: UB-PAD - Paderborn University Library, Heinz Nixdorf MuseumsForum.

Huth, T., Inkermann, D., Wilms, R., \& Vietor, T. (2018). Model-based process engineering - an approach to integrated product system and process modelling. Tag des Systems Engineerings, Berlin, 05.-07. November.

Inkermann, D., Huth, T., Vietor, T., Grewe, A., Knieke, C., \& Rausch, A. (2019). Model-based requirement engineering to support development of complex systems. Procedia CIRP, 84, 239-244.

Kauffeld, S. (2016). Nachhaltige Personalentwicklung und Weiterbildung. Betriebliche Seminare und Trainings entwickeln, Erfolge messen, Transfer sichern (2. Aufl.). Berlin: Springer.

Kauffeld, S., \& Paulsen, H. (2018). Kompetenzmanagement in Unternehmen. Kompetenzen beschreiben, messen, entwickeln und nutzen. Stuttgart: Kohlhammer.

Object Management Group (2017a). OMG systems modeling language (OMG SysML ${ }^{\mathrm{TM}}$ ). http://www.omg.org/spec/SysML/1.5/. Zugegriffen: 8. Febr. 2019.

Object Management Group (2017b). OMG Unified Modeling Language (OMG UML). https://www.omg.org/spec/UML/2.5.1/. Zugegriffen: 8. Febr. 2019.

Ropohl, G. (1975). Systemtechnik - Grundlagen und Anwendung. München: Hanser.

Ropohl, G. (2012). Allgemeine Systemtheorie: Einführung in transdisziplinäres Denken (1. Aufl.). Berlin: edition sigma.

Walden, D.D., Roedler, G. J., Forsberg, K., Hamelin, R. D., Shortell, T. M., \& Kaffenberger, R. (Hrsg.). (2017). INCOSE Systems Engineering Handbuch: Ein Leitfaden für Systemlebenszyklus-Prozesse und -Aktivitäten INCOSE-TP-2003-002-04 2015. München: GfSE e.V. deutsche Übersetzung der vierten Ausgabe

Weilkiens, T. (2014). Systems Engineering mit SysML/UML: Anforderungen, Analyse, Architektur. Bd. 3. Heidelberg: dpunkt.

Wilms, R., Inkermann, D., Cemmasson, V.F., Reik, M., \& Vietor, T. (2019). Distinction of domain-specific and cross-domain linkage types for engineering change management. Proceedings of the Design Society: International Conference on Engineering Design, 1(1), 1125-1134. 


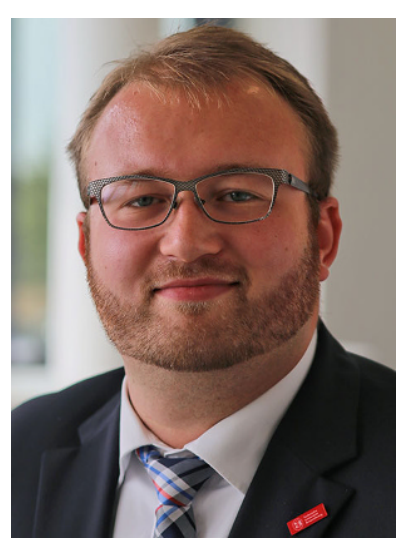

Tobias Huth ist Leiter der Arbeitsgruppe integrierte Produktentwicklung am Institut für Konstruktionstechnik der TU Braunschweig. Er forscht im Bereich des Systems Engineerings und der Produktentwicklungsprozesse.

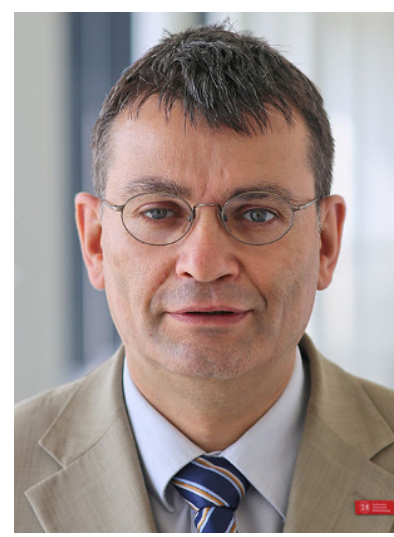

Prof. Dr.-Ing. Thomas Vietor ist Leiter des Instituts für Konstruktionstechnik der TU Braunschweig und Vorstandssprecher des Niedersächischen Forschungszentrums Fahrzeugtechnik. Er forscht u. a. auf den Gebieten des Systems Engineerings, der additven Fertigungsverfahren und des Multi-MaterialLeichtbaus. 